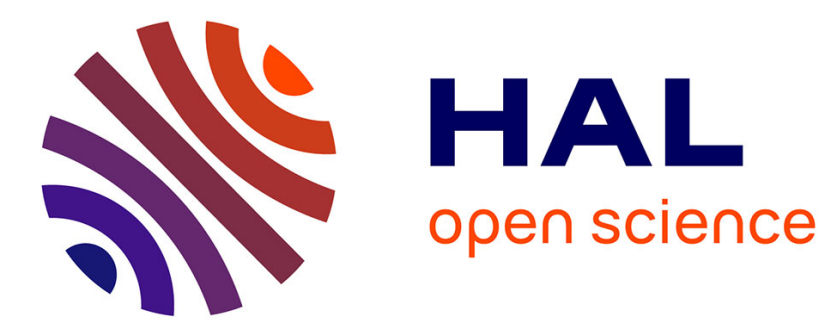

\title{
Du nouveau à propos de l'histoire du complément
}

Bérengère Bouard

\section{To cite this version:}

Bérengère Bouard. Du nouveau à propos de l'histoire du complément. L'information grammaticale, 2008, 117, pp.3-8. hal-01117681

\section{HAL Id: hal-01117681 \\ https://hal.science/hal-01117681}

Submitted on 17 Feb 2015

HAL is a multi-disciplinary open access archive for the deposit and dissemination of scientific research documents, whether they are published or not. The documents may come from teaching and research institutions in France or abroad, or from public or private research centers.
L'archive ouverte pluridisciplinaire HAL, est destinée au dépôt et à la diffusion de documents scientifiques de niveau recherche, publiés ou non, émanant des établissements d'enseignement et de recherche français ou étrangers, des laboratoires publics ou privés. 


\section{Bérengère Bouard \\ UMR 7597 HTL}

\section{Du nouveau à propos de l'histoire du complément}

Deux ouvrages fondateurs ont retracé l'histoire du complément. Le premier, celui de JeanClaude Chevalier (1968) a montré comment la grammaire française centrée sur l'analyse des formes, a évolué vers une grammaire laissant une place à l'analyse syntaxique. Cette évolution passe par la suppression de la déclinaison du nom et la reconnaissance de l'article, ainsi que par l'abandon du régime et l'adoption du complément. Le second, celui d'André Chervel (1977) défend l'idée que la généralisation de la fonction de complément est permise par la seconde grammaire scolaire et le développement d'une pédagogie de l'orthographe. Quarante ans nous séparent du premier ouvrage et trente ans du second. Or, c'est durant cette période que les recherches en histoire et épistémologie des sciences du langage se sont le plus développées. Aujourd'hui, au regard des connaissances nouvelles cumulées dans ce champ, nous pouvons avancer des éléments nouveaux à propos de l'histoire du complément. Notre thèse est que l'émergence de la notion de complément est liée à la déconstruction progressive du modèle propositionnel port-royaliste ${ }^{1}$. La Grammaire générale et raisonnée de Port-Royal (1660) adopte une représentation tripartite de la proposition: sujet-verbe substantif-attribut dans laquelle chaque verbe est décomposé à l'aide du verbe être et du participe présent ; ainsi Pierre vit est équivalent à Pierre est vivant. Cette structure constitue un obstacle à l'émergence de la notion de complément, question où se nouent trois problèmes syntaxiques. Le premier est celui de la décomposition de tout verbe. L'abandon de cette pratique est subordonné à la remise en question de la primauté du verbe substantif dans la proposition. Le second, parallèle au premier, réside dans l'élaboration de la catégorie de verbe transitif. Le troisième correspond à l'intégration d'un constituant nominal en fonction de complément du verbe dans la structure propositionnelle.

\section{La remise en question de la décomposition à l'aide du verbe substantif à partir des années 1820}

La notion de verbe substantif est ancienne ${ }^{2}$ mais le geste marquant de Port-Royal est de lui octroyer un rôle central dans la proposition, impliquant la réécriture être-participe ${ }^{3}$. Le refus de cette pratique est exprimé explicitement à partir des années 1815-1820 par la Société Grammaticale de Paris. Les membres de ce groupe ${ }^{4}$ formulent une nouvelle doctrine grammaticale dont les traits principaux sont une remise en question du système des parties du discours $^{5}$, une redéfinition de la proposition, et le refus de la décomposition du verbe. Les thèses des sociétaires sont exposées dans différentes publications ${ }^{6}$ dont les Annales de grammaire par la société grammaticale de Paris (1818-1820) ${ }^{7}$. Le discours de Vanier, qui se réclame d'une «nouvelle école» (Vanier, 1836, p. 8, p. 34), représente une atteinte importante au modèle propositionnel incluant la décomposition.

\subsection{Vanier}

\footnotetext{
1 Voir la thèse de Bouard, 2007.

2 On la trouve chez Aristote, Priscien, et les Modistes. Cette catégorie est donc transmise aux premiers grammairiens du français avec le classement des cinq « genres » du verbe.

${ }^{3}$ GGR, 1993, p. 110 .

${ }^{4}$ Entre 1815 et 1843 , de nombreux grammairiens et enseignants participent à la Société Grammaticale de Paris fondée par Domergue en 1807 (Auroux, 1983, 1996), tels Lemare, Boniface, Vanier, ou encore Bescherelle.

5 Voir Reuillon-Blanquet, 2005.

${ }^{6}$ Il s'agit des articles du Journal grammatical parus entre 1826 et 1840 (St Gérand, 1996), de dictionnaires grammaticaux (Vanier, Nodier), mais aussi de grammaires ou de traités.

${ }_{7}$ Ces Annales (premier et seul tome) regroupent les procès-verbaux des séances de la Société tenues en 1817 et 1818 .
} 
Vanier refuse la définition de la proposition comme forme linguistique du jugement énoncée par Port-Royal (1827, Traité, Préface, p. 1-3) et élargit son sens à «l'expression d'une pensée » (op. cit., p.6). Parallèlement, le verbe est redéfini comme «la manifestation du mode de la pensée » $(1818$, p. 446). Il n'est plus conçu comme signe de l'affirmation ni outil de liaison entre le sujet et l'attribut (op. cit., p. 501). En d'autres termes, la valeur cohésive et la valeur assertive du verbe dans la proposition sont niées. L'idée du verbe être copule est par ailleurs totalement rejetée dans le Dictionnaire (1836, article «Copule », p.197). Mais les positions ne sont pas homogènes et la retranscription des débats dans les Annales témoigne de la diversité des opinions, la décomposition du verbe étant parfois conservée par Vanier. Ce dernier l'utilise notamment pour distinguer entre les verbes d'état, réécrits à l'aide du participe passé, et les verbes d'action, réécrits à l'aide du participe présent $(1836$, article «Actif », p. 13).

\subsection{Boniface}

Boniface ne rejette pas la définition de la proposition comme l'expression d'un jugement mais il l'élargit en y incluant différentes réalisations phrastiques $\left(1843\right.$, p.2-3) ${ }^{8}$ regroupées sous le terme d' «énonciation ${ }^{9}$. Ainsi, la proposition ne se restreint plus à l'expression de l'affirmation comme dans la définition de la GGR. En outre, si Boniface mentionne le verbe substantif, opposé au verbe attributif (op. cit., p. 3, p. 26), il s'oppose à la décomposition systématique du verbe en être suivi du participe car elle n'est pas jugée équivalente, elle entraîne un changement sémantique notable :

Il ne faut pas croire cependant que le verbe attributif soit réellement composé du verbe être et de l'attribut, dont il n'est pas même l'expression synonyme puisqu'il y a une différence essentielle entre il brille et il est brillant, il aime et il est aimant, etc. (op. cit., p. 3)

Il la considère alors comme une commodité, une convention dans l'analyse :

(..) aussi pour l'analyse de la proposition décompose $\mathrm{t}$ on mentalement il aime par $i l$ est aimant, il brillait par il était brillant, quoique cette décomposition, comme toutes les décompositions, fasse naître un sens différent. (op. cit., p. 26)

Toutefois, Boniface l'accepte lorsque le sujet représenté détient un sens agissant comme dans il brille, il aime, (op. cit., p. 3). Si le grammairien n'adhère pas à la conception de la proposition issue de Port-Royal, la décomposition du verbe lui apparaissant comme un non sens grammatical, il tente pourtant de préserver le modèle initial. En somme, chez ces grammairiens des années 1820-30 participant à la Société Grammaticale ${ }^{10}$, la définition de la proposition comme expression d'un jugement est rejetée et son sens est élargi, de même que les définitions connues du verbe, sont réfutées. Ceci n'est pas nouveau ${ }^{11}$, en revanche ce qui est nouveau, c'est le refus de la décomposition et l'ébranlement par là même de l'importance du verbe substantif dans la proposition. Il s'agit d'un geste fort de positionnement contre cette pratique enseignée et répandue au début du $19^{\text {ème }}$ siècle dans la grammaire française. Cette attitude trouve un écho chez certains grammairiens enseignants comme Perron ${ }^{12}$ qui

\footnotetext{
${ }^{8}$ Les exemples sont : Dieu m'est favorable/ Dieu, soyez moi favorable / Dieu me soit favorable! / Dieu m'est il favorable? (op. cit., p. 2).

9 On lit: «Chacune de ces énonciations, étant l'expression d'un jugement, est une proposition. » (op. cit., p. 3). Ce terme provient de Du Marsais, il reçoit un sens générique mais n'évince pas celui de proposition chez Boniface.

${ }^{10}$ On pourrait aussi mentionner les Bescherelle qui rejettent l'importance du verbe substantif et de la décomposition (1834, Théorie, p. 77) et qui s’opposent à la méthode de Noël et Chapsal (1838).

${ }^{11}$ Girard récuse la définition du verbe de Port-Royal (1747, p. 50-64) et Du Marsais élargit la définition de la proposition (1729-1756, p. 439).

12 Perron, professeur à la faculté des Lettres et secrétaire de l'Académie de Besançon, souhaite « faire du nouveau » dans les classes (1848, p. III, Préface) et défend une réforme de l'enseignement (1851) (voir Bourquin, 2005b).
} 
dénonce l'idée que le verbe être est le «verbe essentiel» auquel tous les autres sont ramenés : "comme si une qualité, une disposition, étaient la même chose qu'un acte ou fait actuel !» (1847, p. VII). Pour lui, la décomposition du verbe en être suivi du participe est « une erreur capitale $»(1851, \text { p. } 15)^{13}$. Mais elle perdurera longtemps. C'est la première nomenclature grammaticale qui l'interdit officiellement en 1910 (Fournier, 2000, Lauwers, 2004). La remise en question de la décomposition et de l'essentialité du verbe substantif est parallèle à l'émergence du verbe transitif, ces deux processus concourant conjointement à la déconstruction du modèle propositionnel port-royaliste.

\section{La délimitation de la catégorie de verbe transitif}

La grammaire française hérite de la tradition latine un classement des verbes reposant sur différents critères mêlés, ainsi le verbe « actif » en latin désigne à la fois le verbe de sens actif, qui a une certaine forme, et qui se construit avec un accusatif ${ }^{14}$. L'histoire de l'émergence de la classe de verbe transitif repose donc sur la séparation de ces critères. Le verbe actif va progressivement être renommé verbe «transitif ${ }^{15}$ et redéfini selon le critère syntaxique.

\subsection{La distinction initiale de Beauzée}

Beauzée formule deux classements des verbes : un classement sémantique reposant sur la signification lexicale distinguant entre verbes actifs, passifs, neutres, et un classement syntaxique reposant sur la possibilité pour le verbe d'être suivi ou non d'un complément (verbe relatif/absolu). Ainsi les verbes relatifs sont des «mots susceptibles de compléments » (1767, p. 45, vol. 2) directs ou indirects comme dans aimer Dieu, craindre sa justice, aller à la ville, revenir de la campagne, passer par le jardin, se repentir de sa faute, commencer à boire, désirer d'être riche, ressembler à quelqu'un (op. cit., p. 50). Silvestre de Sacy, s'inspirant de Beauzée, tente aussi un classement différenciant les niveaux sémantique et syntaxique.

\subsection{Le classement multicritère de Silvestre de Sacy}

Tout d'abord, Silvestre de Sacy distingue, parmi les verbes attributifs (1799, p. 8-9), trois types de verbes selon le sens de l'attribut; les verbes actif, passif ${ }^{16}$ ou neutre ${ }^{17}$. Ensuite, au sein des verbes actifs, il sépare les verbes absolus et relatifs, reprenant la distinction de Beauzée : les verbes absolus «renferment en eux même un sens complet comme je dors, je veille» (op. cit., p. 154-155) alors que les verbes relatifs sont «ceux qui exigent un complément » comme je possède, je vois (op. cit., p. 155). Mais il ajoute une caractérisation puisque parmi les verbes relatifs c'est-à-dire transitifs, il distingue ceux qui se construisent directement ou indirectement: les verbes relatifs suivis d'un complément médiat sont nommés intransitifs, alors que les verbes relatifs suivis d'un complément immédiat ${ }^{18}$ sont nommés transitifs :

Les verbes relatifs gouvernent leurs compléments ou immédiatement ou médiatement. Ceux qui gouvernent leurs compléments immédiatement sont nommés transitifs, les autres intransitifs. (1799, p.156)

Par exemple «lire » est un verbe transitif car il a un complément immédiat comme dans je lis le livre. En revanche, venir est un verbe intransitif car il a un complément médiat commençant par une préposition, comme dans je viens de Rouen. Le vocabulaire de la transitivité est

\footnotetext{
${ }^{13}$ Il écrit justement «pour détruire ces erreurs généralement répandues » (1847, p. VII) et tenter de réformer cette pratique.

${ }^{14}$ Voir Colombat, 1999 et 2006.

${ }^{15}$ Cette caractérisation est aussi ancienne (Colombat, 2003 et à par.).

${ }^{16}$ La définition du verbe passif renvoie plutôt à sa diathèse. Notion que le grammairien tente d'isoler en distinguant trois types de « voix » qui correspondent à autant de «points de vue différents sous lesquels on peut envisager la même action. » (op. cit., p. 146-147). La voix subjective est celle du verbe actif, la voix objective est celle du verbe passif, et la voix neutre est celle du verbe neutre.

${ }^{17}$ Cette classe regroupe des verbes intransitifs mais aussi transitifs indirects.

${ }^{18}$ Cette distinction entre compléments médiat et immédiat provient aussi de Beauzée.
} 
employé en un sens formel ; le qualificatif de transitif désignant la construction directe du verbe, et le qualificatif d'intransitif désignant la construction indirecte du verbe. Cette typologie $^{19}$ représente une tentative innovante pour démêler les critères imbriqués du classement traditionnel. L'ensemble de ces niveaux permet en effet de caractériser séparément le verbe à quatre égards : son sémantisme, sa transitivité, la forme de son complément, et la diathèse. L'effort de Sacy, inspiré par Beauzée, marque le point de départ de l'évolution du classement des verbes jusqu'ici relativement uniforme. Une étape supplémentaire dans l'élaboration des catégories modernes est franchie par Bernard Jullien.

\subsection{Le classement moderne de Bernard Jullien.}

Bernard Jullien ${ }^{20}$ élabore un système de classement des verbes à deux niveaux.

i) Le premier niveau repose sur la notion de voix du verbe. Jullien distingue quatre voix selon la transition ou non de l'action, selon sa direction (du sujet vers le complément ou l'inverse) et les rôles sémantiques des actants ${ }^{21}$. La première voix est nommée simple ou primitive, elle repose sur une transition du sujet vers le complément comme dans j'attache Ferdinand, la seconde est dite pronominée, elle exprime «la station, l'immobilité de l'âme » comme dans je $m$ 'attache à Ferdinand, la troisième se nomme participée, elle consiste en une transition du complément vers le sujet comme dans je suis attaché par Ferdinand, la quatrième est appelée activante elle relève aussi d'une transition sur le complément mais qui ne provient pas du sujet et elle a un sens actif, comme dans je fais attacher Ferdinand (1854, p. 44, p. 47).

ii) Sous la «voix simple et primitive », Jullien distingue cinq sortes de verbes (op. cit., p. 45). Tout d'abord, les verbes sont dits transitifs ou intransitifs selon la présence ou non d'un complément. Le verbe est transitif dans j'attache Ferdinand, je bâtis une maison car «l'idée d'attacher et celle de bâtir sortent du sujet pour tomber sur le complément » (op. cit., p. 45). Le verbe est intransitif dans je marche, je dors, je saigne, je souffre car «les verbes non seulement n'ont pas de complément, mais même ils n'en demandent pas : l'idée reste dans le sujet, il n'y a pas transition » (ibid.). Ensuite, ces verbes transitifs ou intransitifs sont actifs, statifs ou passifs. Les deux caractérisations: syntaxique et sémantique, se combinent; par exemple je marche est intransitif actif, je chante une chanson est transitif actif, je pâtis est passif intransitif, je reçois une visite, je souffre une injure sont passifs transitifs (ibid.). Enfin, le verbe transitif peut être direct ou indirect comme dans il frappe son père : "transitif direct », et il obéit à son père : «transitif indirect ».

Du point de vue des catégories et de la terminologie il s'agit d'une vraie réforme. Jullien refuse les classes traditionnelles des verbes actifs, neutres et surtout passifs et invite à séparer le critère sémantique et le critère syntaxique, qui, superposés depuis trois siècles dans la grammaire française, définissent la classe des verbes actifs :

\footnotetext{
${ }^{19}$ Elle est reproduite dans l'édition de 1809 (Lauwers et Swiggers, 2005).

${ }^{20}$ Bernard Jullien est un savant pédagogue réformateur, connaissant les écrits des Encyclopédistes. Il publie un Cours raisonné (1852) accompagné d'un supplément très intéressant destiné aux enseignants (1854).

${ }^{21}$ On lit : « $1^{\circ}$ l'idée d'attacher sort de moi pour tomber sur Ferdinand, il y a transition, passage du sujet sur le complément $2^{\circ} 1^{\prime}$ idée d'attacher naît et reste en moi $3^{\circ} 1^{\prime}$ idée sort de Ferdinand pour tomber sur moi; c'est une transition du complément sur le sujet $4^{\circ}$ je suis cause que l'idée d'attacher tombe sur Ferdinand. Il y a transition de l'idée sur le complément mais elle ne vient pas immédiatement du sujet. (...) Dans tout cela l'idée d'existence reste la même, l'idée propre du verbe a seule varié, or cette altération dans l'idée propre d'un verbe a reçu le nom de voix : il y a donc quatre voix dans le verbe attacher. » (op. cit., p. 44).
} 
Le mot verbe actif indique deux choses : d'après l'étymologie c'est un verbe dont le sujet fait une action : en ce sens agir, courir, marcher, nager, sont des verbes actifs. Dans une autre acception, le verbe actif est celui qui prend un complément direct : en ce sens faire, prendre, recevoir, souffrir, sont actifs quoique les deux derniers n'indiquent pas du tout une action faite, mais bien une action reçue par le sujet (...) ainsi quoi que l'on fasse le mot de verbe actif, tant qu'il ne sera pas autrement déterminé, entraînera toujours une confusion d'idées, qui plus tard se traduira en difficultés très sérieuses pour les enfants. (1854, p. 12)

Il renomme le verbe actif, transitif; les verbes transitifs étant «ceux qui prennent des compléments » (ibid.). En même temps, Jullien redistribue les verbes neutres (op. cit., p. 14) : il fonde la nouvelle classe des intransitifs et intègre les verbes prépositionnels aux transitifs indirects (je pense à vous, je vais à Versailles, je viens de Paris). Redéfinir et renommer le verbe selon sa relation sémantico-syntaxique avec le complément revient à prendre en compte un autre modèle propositionnel que le schéma attributif ; la structure transitive. Celle-ci inclut un complément à la suite du verbe. Auparavant, les grammairiens de l'âge classique ont tenté de diverses manières d'infléchir le modèle attributif univoque des Messieurs $^{22}$. Mais c'est Beauzée le premier qui accorde une définition au complément et construit une typologie spécifique, dans 1'Encyclopédie Méthodique (1782-1786) et dans sa Grammaire Générale $(1767)^{23}$.

\section{L'intégration du complément du verbe dans la structure propositionnelle au $19^{\text {ème }}$ siècle}

Après Beauzée le complément suit un parcours compliqué, amplement lié à l'évolution de la grammaire générale au $19^{\text {ème }}$ siècle.

\subsection{Les positions des grammairiens au tournant des $18^{\text {ème }}$ et $19^{\text {ème }}$ siècles}

Au tournant des $18^{\text {ème }}$ et $19^{\text {ème }}$ siècles, le complément n'est pas unanimement adopté. Prenons deux exemples parlants. Domergue ${ }^{24}$ conserve la définition de la proposition comme expression du jugement ainsi que la structure propositionnelle tripartite (Domergue, 1798, p. 11$)^{25}$ mais il y ajoute le « complément » comme quatrième constituant :

Il y a donc, outre les trois parties logiques, essentielles, une quatrième partie que j'appelle complément. $\left(1798\right.$, p. 16) ${ }^{26}$.

Le complément est considéré comme une expansion grammaticale des autres parties de la proposition (hormis du verbe être). Domergue choisit volontairement le terme et la notion de complément qu'il défend (op. cit., p. 22-24), et il utilise une nouvelle terminologie : les compléments sont dits éloignés ou prochains ${ }^{27}$, et direct ou indirect (op. cit., p. 18-20), caractérisations qui se combinent. Mais Domergue apparaît comme un innovateur isolé. En effet, l'adoption du complément n'est visible que chez certains grammairiens minoritaires, lecteurs de l'Encyclopédie. Ce que l'on observe majoritairement, c'est la conservation de la notion de régime, en coexistence avec des mentions du complément comme chez Loneux

\footnotetext{
${ }^{22}$ Buffier avec le modificatif (Roelandt et Swiggers, 1990, Bouard, 2008a), Girard avec un système à sept fonctions, ou encore Du Marsais avec le déterminant, intègrent des compléments du nom ou du verbe dans la proposition, à l'aide de différents termes et de différentes notions (Bouard, 2008b).

${ }^{23}$ Voir Auroux, 1973 et 1979, Swiggers, 1989.

${ }^{24}$ Urbain Domergue, professeur de grammaire générale à l'Ecole des Quatre nations, membre de l'Institut de langue française, est une figure importante de la fin du $18^{\text {ème }}$ siècle et du début du $19^{\text {ème }}$ siècle, c'est lui qui fonde le Journal de langue française en 1784 (Auroux, 1982, Dougnac, 1981).

${ }^{25}$ Domergue désigne les trois composantes de la proposition à l'aide d'une nouvelle terminologie issue du le verbe juger en latin : judicande - judicateur-judicat, et il pratique systématiquement la décomposition.

${ }^{26}$ L'exemple est : la culture (judicande) de l'esprit (complément) est convenant (judicateur + judicat) aux personnes (complément) des deux sexes (complément).

${ }^{27}$ Le complément dit prochain est avant tout conçu comme essentiel par Domergue : nous déjouerons - avant six mois (complément éloigné) - les projets (complément prochain par rapport au verbe) - de nos ennemis (complément prochain par rapport à projets).
} 
(1799), ou Lévizac (1809). On relève donc l'existence de nombreux systèmes syntaxiques mêlant les deux terminologies de complément et de régime, comme chez Thiebault Dieudonné. Dans sa Grammaire philosophique (1802) il conserve la structure tripartite de Domergue et y ajoute des «parties accidentelles, ou circonstancielles et accessoires » (1802, p. 11, vol. 2) nommées compléments :

Toutes ces idées appelées à la suite des idées générales ou relatives sont donc des additions souvent nécessaires pour compléter le sens de celles auxquelles on les joint : aussi leur a-t-on donné le nom de complément. Les compléments d'une idée ou d'un mot sont de cette sorte, d'autres idées ou d'autres mots qu'on y rapporte ou que l'on met sous sa dépendance, pour en expliquer ou déterminer, spécifier, arrondir et compléter le sens. (op. cit., p. 15).

Le grammairien distingue les compléments identique et relatif (op. cit., p. 17) mais aussi deux classes de régimes : les régimes simples ou directs, et composés ou obliques (op. cit., p. 18) qui sont renommés à l'aide de la terminologie de Girard et de Beauzée lorsqu'ils se rapportent au verbe (régime objectif et primitif / régime terminatif ou secondaire). Cette position hésitante témoigne de la difficulté des grammairiens à décrire le syntagme complétant le verbe dans le modèle propositionnel port-royaliste au tournant des $18^{\text {ème }}$ et $19^{\text {ème }}$ siècles. La version condillacienne de la grammaire générale entretient le modèle attributif (Condillac, 1775, Grammaire, p. 102-103 $)^{28}$ mais c'est surtout sous l'influence de l'école idéologique et de l'enseignement de la grammaire générale dans les écoles centrales ${ }^{29}$ que le modèle se durcit, engendrant un oubli provisoire du complément.

\section{2. L'oubli du complément avec l'école idéologique au début du $19^{\text {ème }}$ siècle}

La doctrine de l'idéologie ou "science des idées » est développée par Destutt de Tracy dans les quatre volumes de ses Eléments d'idéologie (1817). Le grammairien conserve un modèle propositionnel tripartite classique (Grammaire, 1817, p. 26) dans lequel l'élément essentiel de la proposition reste le verbe au mode indicatif (op. cit., p. 43-44, p. 59). Par ailleurs, le verbe est conçu comme un type d' adjectif $^{30}$, tous les verbes étant composés de la forme étant, adjectif exprimant l'existence, et d'un autre adjectif ou modificatif, qui serait incomplet tout seul (op. cit., p. 64). La question de la transitivité est donc évacuée, comme chez Condillac ${ }^{31}$, et lorsque la fonction de complément est évoquée elle n'est pas mise en relation avec le verbe, il s'agit soit du «complément d'un sujet» (op.cit., p. 73), soit du complément de «l'attribut » ou de «l'adjectif »:

Lorsqu'il indique le besoin d'un complément je désire, je tâche... ce n'est pas comme verbe qu'il a besoin de ce complément mais comme adjectif, ce qu'on appelle régime est le complément de la signification de l'adjectif. (op. cit. , p.93)

De façon générale, chez les Idéologues, l'élaboration des catégories fonctionnelles est délaissée, le débat étant centré sur les classes de mots. La structure propositionnelle évolue alors vers le schéma suivant : sujet-est-adjectif-complément, où l'adjectif désigne en fait le

\footnotetext{
${ }^{28}$ Condillac décrit les phénomènes de complémentation à l'aide de deux autres notions : «les accessoires » (1775, Grammaire, p. 115, 117, 125, 2ème partie, Syntaxe, p.297) et les « modifications » (Art d'écrire et de raisonner, p. 31).

${ }^{29}$ Les Ecoles Centrales sont créées par décret du 7 ventôse an III (25 février 1795) rendu sur le rapport de Lakanal et De Tracy (16 décembre 1794) et elles sont officiellement instituées le 3 brumaire an IV (25 octobre 1795). Il y en a une par département, le programme est assuré par le professeur de grammaire générale ou de philosophie et doit suivre une série de circulaires rédigées par Destutt de Tracy et agréées par le Ministre. En 1803, la Commission de l'Instruction Publique est dissoute et les écoles centrales sont supprimées.

${ }^{30}$ Les verbes ne sont pas conçus comme des mots d'ordre supérieur, ils sont considérés comme des adjectifs, et l'adjectif comme un verbe: «les adjectifs proprement dit sont des verbes mutilés et les verbes sont des adjectifs entiers » (op. cit., p. 58), de même que chez Condillac le verbe provient de l'adjectif.

31 Destutt de Tracy récuse les classements du verbe et considère tous les verbes comme des verbes d'état (op. cit., p. 84), Condillac lui ne distingue qu'entre les verbes d'état et les verbes d'action.
} 
participe, et le complément est le complément de l'adjectif et non du verbe être, qui est complet par lui-même.

\subsection{L’ambivalence des grammaires générales tardives au milieu du $19^{\text {ème }}$ siècle}

Les grammaires générales du milieu du $19^{\text {ème }}$ siècle conservent majoritairement le modèle propositionnel tripartite de Port-Royal, la décomposition apparaissant comme une évidence, mais le modèle peut aussi être augmenté. L'intégration du complément est très nette chez Caillot qui se présente comme un vrai disciple de Vanier (Caillot, 1838, p. 96). Ainsi, il s'élève contre la définition de la proposition comme expression du jugement, qu'il juge être « une absurdité » (op. cit., p. 130), et préfère identifier la proposition à «l'expression d'une pensée » (op. cit., p. 122). L'assimilation de la proposition à une phrase nécessairement assertive par Port-Royal ne convient plus du tout aux auteurs de grammaires générales et son sens est élargi aux différents types de phrases. Caillot adopte le complément et différencie le complément direct qui « reçoit directement l'action faite par le sujet » comme dans Pierre aime une femme (vol. 2, p. 388), et le complément indirect qui « reçoit indirectement cette action » comme dans Pierre court après une femme (op. cit., p. 389). D'autres grammairiens conservent le régime comme seule fonction (Jonain-1835-) ou comme synonyme du complément (Poitevin -1858-, Bel -1829-), ou bien emploient d'autres termes comme celui de «modificatif» (Montémont-1845). Ce dernier distingue les modificatifs qui sont absolus comme dans le sage est heureux, relatifs objectifs comme dans la vertu fait le bonheur, ou relatifs circonstantifs comme dans l'homme est heureux par la vertu, Les Tarquins furent bannis de Rome. Les deux peuvent se combiner, comme dans le sage trouve son bonheur dans la vertu. (Montémont, 1845, p. 122-123). Globalement, la position des auteurs des grammaires générales du milieu du $19^{\text {ème }}$ siècle est de respecter la tradition port-royaliste tout en prenant en compte l'invention de Beauzée (qui aussi est généralisée chez Boniface ou Bescherelle). Il en résulte une certaine ambivalence. La survivance d'une tradition de grammaires générales au 19ème siècle explique la persistance du modèle ancien, modèle qui est sérieusement remis en question par ailleurs autour des années 1820 par la Société grammaticale.

\section{Conclusion}

L'histoire du complément tresse trois fils. Les deux premiers concernent la définition et le statut du verbe dans la proposition. La déconstruction du modèle propositionnel passe premièrement par l'abandon de la primauté du verbe substantif et deuxièmement par la réintroduction, parallèle, de la catégorie de verbe transitif dans la structure propositionnelle. Le troisième problème correspond à la reconnaissance d'un constituant nominal en fonction de complément du verbe. L'observation concomitante de ces trois problématiques permet aujourd'hui d'identifier d'autres étapes dans l'histoire de la construction de la notion de complément. Chervel a bien montré comment la première grammaire scolaire naît en 1823 avec la Nouvelle grammaire française de Noël et Chapsal qui impose le modèle propositionnel ternaire de la grammaire générale à l'école (Chervel, 1977, p. 97-98). Cet ouvrage diffuse une analyse logique de la proposition comprenant la décomposition du verbe (Chevalier, 1979, Chervel, 1977, p. 100, p.156-158). C'est avec le mouvement de la seconde grammaire scolaire, entre les années 1860 et 1880 (op. cit., p. 140, 161-162), que ce modèle est mis à mal (Chervel, 1977, p. 136), ce qui s'accompagne de la généralisation du complément (op.cit., p. 118, p. 178). L'utilisation du complément et les distinctions liées (direct/indirect) se justifient en effet par la nécessité de savoir accorder le participe (op.cit., p. 102-103). Nous adoptons une position différente :

i) Premièrement, il est clair que le processus de déconstruction du modèle propositionnel a débuté bien avant. Il se réalise selon nous en deux étapes. C'est tout d'abord la doctrine de la Société Grammaticale qui assène le premier coup au modèle initial à partir de 1818, dans les Annales. Cette position est prolongée jusqu'au milieu du siècle par diverses figures comme 
Bescherelle (1834), Caillot (1838), Perron (1847, 1848, 1851). La seconde étape décisive correspond au mouvement de réforme défendu par Bernard Jullien en 1854, qui accorde une place au verbe transitif dans la proposition. La structure tripartite n'est plus érigée en modèle, même si la décomposition et l'opposition entre le verbe substantif et les autres verbes sont conservés longtemps.

ii) Deuxièmement, sur le plan épistémologique, il est nécessaire de rapporter l'histoire du complément à l'évolution de la représentation de la proposition dans la grammaire en général, et à l'évolution du mouvement qui a formulé les coordonnées du modèle originel : celui de la grammaire générale, qui connaît des prolongements au $19^{\text {ème }}$ siècle (Bourquin, 2005a). Il conviendrait cependant d'interroger plus longuement les liens entre les grammaires scolaires et les grammaires autres.

Par ailleurs, l'observation d'un corpus plus large, des premières grammaires du français aux grammaires générales tardives, permet de saisir des mouvements d'ensemble et les évolutions parallèles de différentes notions (le complément, la voix verbale, le verbe transitif).

Bérengère Bouard

Berengere.bouard@linguist.jussieu.fr

UMR 7597 Histoire des Théories Linguistiques

Université de Caen

\section{Références bibliographiques primaires}

ARNAUld A. \& LANCELOT C., 1993, Grammaire générale et raisonnée, reproduction de l'édition de Paris 1845, Genève : Slatkine Reprints.

BEAUZEE N., 1767, Grammaire Générale, ou Exposition raisonnée des éléments nécessaires du langage, pour servir de fondement à l'étude de toutes les langues, Paris : J. Barbou, 2 vol.

- 1782-1786, Article «Complément» de l'Encyclopédie Méthodique Grammaire et Littérature, Paris-Liège : Panckoucke, 3 vol.

BEL, A., 1829, Grammaire françoise élémentaire et raisonnée comprenant les principes de la grammaire générale, la syntaxe, la construction, l'orthographe absolue, celle d'usage et la versification, Paris : Hachette, Delalain.

BESCHERELLE, L.-N., et H.-H., 1834, Grammaire nationale, Paris ; Bourgeois-MAZE, 2 vol. - 1838, Réfutation complète de la Grammaire de Noël et Chapsal, Paris : Bourgeois-Maze.

BONIFACE, A., 1843 (1829), Grammaire Française, Méthodique et Raisonnée, Paris : Delalain.

BUfFIER, le Père C., 1709, Grammaire françoise sur un plan nouveau, Paris : N. le Clerc.

CAILlot, N., 1838, Grammaire générale et philosophique et critique de la langue française, Paris : boulevard du Temple, 2 vol.

Condillac, E. B. , Abbé de, 1775, Grammaire (tome 1 du Cours d'Etudes pour l'instruction du Prince de Parme), Art d'écrire et de raisonner (tome 2), Parme : Imprimerie royale.

Destutt, Comte de Tracy, A. L. C., 1817, Eléments d'idéologie, $1^{\text {ère }}$ partie Idéologie proprement dite, $2^{\text {nde }}$ partie : Grammaire, Paris : Courcier, 1970, Paris : Vrin.

Domergue, F. U., 1798-1799, Grammaire générale analytique..., Paris : impr. de C. Houel.

Du MARSAIS, C. Ch., 1729-1756, Les véritables principes de la grammaire et autres textes, Douay-Soublin F. éd., Paris : Fayard, 1987.

GIRARD, Abbé G., 1747, Les vrais principes de la langue françoise ou la parole réduite en méthode, conformément aux lois de l'usage, Paris : Le Breton, 2 vol.

JoNAIN, P., 1835, Grammaire générale contenant les grammaires particulières et complètes du latin et du français parallèlement démontrées, Paris : Aimé André.

JulliEn, B., 1852, Traité complet de langue française Cours raisonné de langue française deuxième degré, Paris : Hachette. 
JULLIEN B., 1852-54, Explication des principales difficultés de l'enseignement de la grammaire (in Cours raisonné), dans le Traité de Grammaire, Paris : Hachette.

LEVIZAC, J.-P.-V. Lacoutz, Abbé de, 1809 (1797), L'Art de parler et d'écrire correctement la langue française, ou Grammaire philosophique et littéraire de cette langue, à l'usage des Français ou des Etrangers qui désirent en connaître à fond les principes, les beautés et le génie, Paris : Rémont.

LONEuX, E., 1799, Grammaire Générale appliquée à la langue française, Liège : L. Bassenge.

MONTEMONT, A., 1845, Grammaire générale ou philosophie des langues présentant l'analyse de l'art de parler, considérée dans l'esprit et dans le discours, au moyen des usages comparés des langues hébraïques, grecque, latine, allemande, anglaise, italienne, espagnole, française et autres, Paris : Moquet, 2 tomes.

NODIER, Ch., et BOISTE, 1834, Dictionnaire universel de la langue française, Paris : Lecointe et Pougin.

NoËL, F.-J.-M. ; ChAPSAL, Ch.-P., 1823, Nouvelle Grammaire Française, Paris : Nyon Jeune. PERRON F., 1847, Elements de grammaire générale ou Nouveaux principes de grammaire française, Paris : Didot frères.

PERRON F., 1848, Nouveaux principes de grammaire française rédigés d'après les éléments de grammaire générale du même auteur, Paris : Didot, Chamerot, Lecoffre, Besançon : Outhenin Chalandre fils.

PERRON F., 1851, Essai d'une réforme de l'enseignement grammatical présenté à l'Académie française et à Mr le Ministre de l'Instruction, Paris : Désobry et Magdeleine, Besançon : Veuve Ch. Deis.

PoITEVIN, M. P., 1858, Grammaire générale et historique de la langue française présentant l'étude et l'analyse de la formation, des développements et des variations de notre idiome national, dans Cours théorique et pratique de langue française, Paris : Bureaux du magasin pittoresque.

Silvestre De SACY, A.-I., 1799, Principes de Grammaire Générale mis à la portée des enfants et propres à servir d'introduction à l'étude de toutes les langues, Paris : Fuchs.

Thiebault D., 1802, Grammaire philosophique ou la métaphysique, la logique et la grammaire, réunies en un seul corps de doctrine, Paris, 2 tomes, Impression en fac similé, introduction par Daniel Droixhe, Friedrich Frommann Verlag Günter Holzboog KG, Stuttgart-Bad Cannstatt, 1977.

VAnier, V.-A., Lemare, Butet, Perrier, ScotT De MARTINVILle, etc, 1818-1820, Annales de grammaire par la Société Grammaticale de Paris, tome 1er, Paris : Béchet.

VANIER, V.-A., 1827, Traité d'analyse logique et grammaticale, Paris : Garnier.

VANIER, V.-A., 1836, Dictionnaire grammatical, critique et philosophique de la langue française, Paris : L'Auteur.

\section{Références bibliographiques secondaires}

AUROUX, S., 1973, "L'Encyclopédie », "Grammaire » et "Langue » au XVIII siècle. Edition présentée et commentée des articles «Grammaire » et «Langue » de 1'Encyclopédie ou dictionnaire raisonnée des sciences, des arts et des métiers, rédigés par Nicolas Beauzée et Jacques Philippe Augustin Douchet, Tours : Mame. Coll. Série bleue, Linguistique, 3. -1979, La sémiotique des Encyclopédistes. Essai d'épistémologie historique des sciences du langage, Payot : Paris.

- et S., Dougnac, F., Horde, T., 1982, «Les premiers périodiques linguistiques français (17841840) », Histoire Epistémologie Langage, IV-1, p. 117-132.

- 1983, «La première Société de Linguistique - Paris, 1837 », Historiographia Linguistica, John Benjamins, X, n 3, pp. 241-266. 
-1996, «Langue, Etat, Nation : le modèle politique», in P. Sériot (éd.) : Langue et nation en Europe centrale et orientale, du 18ème siècle à nos jours, Cahiers de l'ILSL nº 8, Univ. de Lausanne, p. 1-20.

BOUARD, B., 2007, Structure de la proposition et construction verbale : régime, complément et transitivité dans les grammaires françaises, 1651-1863, thèse de doctorat, dir. S. Auroux, Univ. Paris 7, Lille : ARDT.

- 2008a à par., Proposition et complément dans la grammaire française : l'histoire du modificatif (1709-1843), Actes du colloque «La structure de la proposition histoire d'un métalangage », 5-7 octobre 2006, Cahiers de 1'I.L.S.L. n²4, Univ. de Lausanne.

- 2008b à par., L'invention terminologique et conceptuelle du complément dans la grammaire française 1660-1863, ColDoc 2007 UMR 7114 MoDyCo Université Paris X Nanterre, «Le vocabulaire scientifique et technique en sciences du langage ».

BOURQUIN, J., 2005a, Les prolongements de la grammaire générale en France et dans les pays francophones au XIXe siècle (1802-1870), colloque de Besançon, 19-21 septembre 2002, Besançon : Presses universitaires de Franche-Comté, introduction, introduction, p. 7-14.

- 2005b, «Les éléments de grammaire générale de François Perron (1847)», dans Bourquin, J. (coord.), Les prolongements de la grammaire générale en France et dans les pays francophones au XIX ${ }^{e}$ siècle (1802-1870), colloque de Besançon, 19-21 septembre 2002, Besançon : Presses universitaires de Franche-Comté, p.181-200.

CHERVEL, A., 1977, Et il fallut apprendre à écrire à tous les petits français. Histoire de la grammaire scolaire, Paris : Payot.

CHEVALIER, J.-C., 1968, Histoire de la syntaxe : naissance de la notion de complément dans la grammaire française, 1530-1750, Genève : Droz [réédition : Paris, Champion, 2006]. -1979, «Analyse grammaticale et analyse logique : esquisse de la naissance d'un dispositif scolaire », Langue française 41, p. 20-34.

COLOMBAT, C., 1999, La grammaire latine en France à la Renaissance et à l'âge classique : théories et pédagogie, Grenoble : ELLUG.

- 2003 «La problématique de la transitivité dans la tradition grammaticale latine quelques jalons de Priscien aux premiers Humanistes », Histoire Epistémologie Langage, XXV-1, p. 153-174.

- 2006, «Les 'accidents' du verbe, les genera verborum et le subjonctif dans les premières grammaires françaises » dans Colbus, J.-C., Hebert, B. (dir.), Les outils de la connaissance : enseignement et formation intellectuelle en Europe entre 1453 et 1715, Saint-Étienne : Publications de l'Université de Saint-Étienne, p. 95-116.

-à par., «Les fondements sémantiques de la transitivité : à la recherche de la personne », dans Van Raemdonck, D., Rosier, L., Evrard, I. (éd.), Actes du colloque Représentations du sens linguistique III, Bruxelles, 3-5 novembre 2005.

DOUGNAC, Françoise, 1981, F.U. Domergue. Le Journal de la langue française et la néologie lexicale (1784-1795), ENS de Saint-Cloud.

FOURNIER, J.-M., 2000, « Â propos de la syntaxe dans les grammaires scolaires à partir de 1910 : la théorie classique de la proposition et des compléments », Etudes de Linguistique appliquée, 118, p. 197-210.

LAUWERS, P., 2004, La description du français entre la tradition grammaticale et la modernité linguistique : étude historique et épistémologique de la grammaire française entre 1907 et 1948, Louvain - Paris - Dudley : Peeters, Orbis Supplementa 24.

- et SWIGGERS, P., 2005, «Silvestre de Sacy et la structure de la proposition», dans Bourquin, J. (coord.), Les prolongements de la grammaire générale en France et dans les pays francophones au XIX siècle (1802-1870), colloque de Besançon, 19-21 septembre 2002, Besançon : Presses universitaires de Franche-Comté, p. 61-77. 
RABY, V., 2000, La proposition dans la Grammaire Générale française (1666-1803), thèse de doctorat dir. S. Auroux, Univ. Paris 7 Denis Diderot, Lille : ARDT.

REUILLON-BLANQUET, M., 2005, "Vive controverse autour du système binaire et du système trinaire Lemare, Destutt de Tracy, Vanier, Michel, Lemeneur-Doray », dans Bourquin, J. (coord.), Les prolongements de la grammaire générale en France et dans les pays francophones au XIXe siècle (1802-1870), colloque de Besançon, 19-21 septembre 2002, Besançon : Presses universitaires de Franche-Comté, p. 151-165.

ROELANDT, J. SWIGGERS, P., 1990, « La modification comme relation sémanticosyntaxique chez Claude Buffier », Travaux de linguistique et de philologie, 28, p. 64-70.

SAINT-GERAND, J.P., 1996, «L'exemple d'une revue : le journal grammatical et didactique de la langue française (1826-1840) » Travaux de linguistique, 33, p. 91-114.

SWIGGERS, P., 1989, «Structure propositionnelle et complémentation dans l'histoire de la grammaire : la théorie de Beauzée (1767) », Lingua e stile, 24, p. 391-407. 